\title{
AVAILABLE TRANSFER CAPABILITY CALCULATION WITH POST-CONTINGENCY GENERATION RESCHEDULING/LOAD CURTAILMENT
}

\author{
W.X. Li, Z.M. Li \\ Dept. of Electrical Engineering \\ Harbin Institute of Technology \\ P.R.China (lwx1121@sina.com)
}

\begin{abstract}
The available transfer capability (ATC) is an important index indicating the remaining transfer capability in the physical transmission network for further commercial activity above existing commitments. In this paper, ATC mathematical model considering post-contingency generation rescheduling and load curtailment is first formulated. Benders decomposition method is then used to partition the ATC model above into a base case master problem and a series of independent subproblems relevant to various contingencies. Finally, an improved parallel solution scheme is employed to improve the convergence. Numerical results on a 4-bus test system show clearly the effectiveness of the presented method and necessity of considering post-contingency generation rescheduling and load curtailment in calculating ATC.
\end{abstract}

\section{INTRODUCTION}

The power industry is in the process of restructuring from a vertically regulated industry to a partially deregulated one. Around the world, available transfer capability (ATC), which is required to be posted on open access same-time information system (OASIS), is becoming an important index indicating the remaining transfer capability in the physical transmission network for further commercial activity above existing commitments, and also an important issue related to congestion management because it can provide useful information for all the market participants so that they can arrange the transactions better to prevent congestion from happening. Therefore, ATC calculation is a common issue of concern to both system planners and operators.

In the new electricity market environment, transmission grids are going to be operated closer and closer to their limits. So, the security and reliability of the transmission system are of most significance and of great concern. They are becoming ever more challenging due to the voluminous amount of transactions traversing the transmission network in various directions. Consequently, the effect of the system security criterion on the transfer capability has

\author{
Y.X. Ni, F. F. Wu \\ Dept. of E.E.E \\ The University of Hong Kong \\ Hong Kong SAR
}

to be addressed bluntly. However, the consideration of the system security criterion, without corrective control, generally makes the ATC too conservative and leads to a costly and inefficient use of the network due to the little contingency probability, which don't meet the market mechanism. It is therefore necessary to consider post-contingency corrective actions, such as phase shifters, tap transformer, FACTS devices, generation rescheduling and load curtailment, etc., in calculating $\mathrm{ATC}^{[1-2]}$.

Mathematically, ATC is defined as total transfer capability (TTC) less existing transmission commitments, less the sum of transmission reliability margin (TRM) and capacity benefit margin $(\mathrm{CBM})^{[3]}$. Among these factors determining ATC values, TTC is a very important one and is defined as the amount of electric power that can be transferred over the interconnected transmission network in a reliable manner, which meets a prespecified set of pre-and-post-contingency system conditions. Although TRM and CBM are also important parts of ATC, however they can be considered separately from TTC calculation. TTC will therefore be addressed in this paper as the basis for ATC calculation assuming TRM and CBM are available.

Linear method based on DC power flow was presented to calculate ATC in [4]. Because of the relative ease coupled with the mild computational burden involved in computing those distribution factors used, it have found widespread applications and development in the power industry. However, since those factors are based on dc load flow ignoring voltage and reactive power effects as well as system nonlinearity, they might lead to unacceptable error especially in a stressed system with insufficient reactive power support and voltage control. Continuation power flow (CPF) that incorporates the effects of the reactive power flows, voltage limits and voltage collapse as well as the traditional thermal loading effects is a popular tool to calculate ATC ${ }^{[5]}$. Undoubtedly CPF is an important step further as compared with the dc load flow based method because it takes system nonlinearity and voltage-reactive power aspects into account. But, since CPF increases the 
loading factor only along certain direction without considering control effects, it may give conservative ATC results. Besides, security constrained OPF method ${ }^{\left[{ }^{6}\right]}$ has been used to solve the static security constrained ATC (SSC-ATC) problem. However, the correct representation of security constraints (and even more so if post-contingency actions are to be taken into account) may cause a great increase of orders of magnitudes in problem size.

In this paper, Benders decomposition ${ }^{[7]}$ is applied for dealing the SSC-ATC problem incorporating the postcontingency generation rescheduling and load curtailment. The SSC-ATC problem is cast as a mathematical programming problem where the objective function is to maximize the transfer power between specific generator(s) and load(s) subject to the constraints of load flow equations and system operating limits. ' $\mathrm{N}-1$ ' security criterion is used with contingency list available. Benders decomposition breaks down the original problem into two levels: the master level (master problem) and the slave level (subproblems). Each contingency can be incorporated into a subproblem, which generates corresponding security bounds to be turned over to the master problem for consideration via a Benders cut. Using an iterative algorithm, Benders cuts corresponding to various contingencies are introduced concurrently to the master problem until the final solution is reached.

This paper is arranged as follows. In Section II, the formulation of SSC-ATC calculation incorporating post-contingency generation rescheduling and load curtailment is presented. Section III applies Benders decomposition to SSC-ATC calculation and forms the corresponding mathematic model, and an improved parallel solution scheme is presented in section IV. Numerical results and conclusions are given in sections $\mathrm{V}$ and $\mathrm{Vl}$, respectively.

\section{FORMULATION OF THE SSC-ATC PROBLEM}

SSC-ATC is the maximum amount of power transferable over the entire transmission network from an injection (sending) node/area denoted as supply generator set $S$ to an extraction (receiving) node/area denoted as receiving load set $R$ without violating any of the pre-and-postcontingency static security constraints. Usually, SSCATC calculation only takes into account the line thermal limits and the voltage limits. The first incurred limit among them sets the final SSC-ATC value. Some assumptions are made for the formulation as follows:
- The base case power flow of the system is feasible and corresponds to a stable and secure operating point.

- The system has sufficient damping torque to keep itself within the steady state stability limit.

- The load and generation patterns vary slowly so that the system dynamic and transient stability is not jeopardized

- Bus voltage limits are reached before the system reaches the nose point and loses voltage stability.

Under these assumptions, system constraint violations will be transmission line flow limits, voltage deviation limits and generator active and reactive power limits under pre-and-post-contingency conditions, i.e. the SSC-ATC calculation incorporating post-contingency generation rescheduling and load curtailment is our target at this stage. The ATC calculation incorporating transient stability will be published in subsequent papers.

Suppose that $h$ is the index of the transaction whose TTC will be calculated. Thus, the objective function of this problem is the TTC value for transaction $h$, which can be obtained as the final generation power summation of this transaction.

Assuming SSC-ATC to be evaluated is between generator bus set $S$ and load bus set $R$, the detailed SSCATC formulation incorporating generation rescheduling and load curtailment can be cast as a mathematical programming problem taking the format below:

$$
\operatorname{Max} \sum_{k \in S} P_{h, k x}
$$

s.t.

$$
\begin{aligned}
& P_{g i}^{p}-P_{l i}^{p}-V_{i}^{p} \sum_{j=1}^{k_{q}} V_{j}^{p}\left(G_{i j}^{p} \cos \theta_{i j}^{p}+B_{i j}^{p} \sin \theta_{i j}^{p}\right)=0 \\
& Q_{k i}^{p}-Q_{i i}^{p}-V_{i}^{p} \sum_{j=1}^{N_{B}} V_{j}^{p}\left(G_{i j}^{p} \sin \theta_{i j}^{p}-B_{i j}^{p} \cos \theta_{i j}^{p}\right)=0 \\
& P_{h, g k}^{\text {min }} \leq P_{h, g h}^{p} \leq P_{h, g k}^{\max }, k \in S \\
& Q_{g m}^{\min } \leq Q_{g m}^{\mu} \leq Q_{g m}^{\max }, m \in N_{G} \\
& P_{h, l d}^{h} \leq P_{h, l d}^{0} \leq P_{h, l d}^{\max }, d \in R \\
& V_{i}^{\text {min }} \leq V_{i}^{p} \leq V_{i}^{\text {nax }}, i=1,2, \ldots, N_{B} \\
& \left|I_{i j}^{p}\right| \leq I_{i j}^{\max }, i, j=1,2, \ldots, N, i \neq j \\
& P_{n . l d}^{h}=P_{n, d d}^{p}, n \neq h \\
& P_{n, g k}^{b}=P_{n, g k}^{r}, n \neq h \\
& P_{h, d}\left(u_{0}\right)-P_{h, d d}^{p}\left(u_{p}\right) \leq \Delta P_{h, d d}^{p}, d \in R \\
& \left|P_{h, g k}\left(u_{0}\right)-P_{h, g k}^{p}\left(u_{p}\right)\right| \leq \Delta P_{h, g k}^{p}, k \in S \\
& p=0 \text { (nomal case) } \\
& p=1,2, \ldots, N_{C} \text { (contingency case) }
\end{aligned}
$$


Where:

$P_{g i}$ is the real power generation supply at bus $i$,

$P_{i j}$ is the actual real load demand at bus $i$,

$Q_{\mathrm{g} i}$ is the reactive power generation supply at bus $i$,

$Q_{l i}$ is the actual reactive load demand at bus $i$,

$V_{i}$ is the voltage magnitude and angle respectively at bus $i$,

$\theta_{i j}$ is the angle difference between the voltages at buses $i$ and $j$,

$\left(G_{i j}+j B_{i j}\right)$ is the $i j^{\text {th }}$ element of the bus admittance matrix,

$P_{h, g k}^{\max }$ and $P_{h, k k}^{\min }$ are the active power limits for generator $k$,

$Q_{g m}^{\max }$ and $Q_{g m}^{\min }$ are the reactive power limits for generator $m$,

$P_{h, d t}^{\max }$ and $P_{l d}^{b}$ are the upper limit and the base value of real

power load at bus $d$,

$P_{g k}^{h}$ is the base value of real power generation for generator $k$,

$\left|I_{i j}\right|$ and $I_{i j}^{\max }$ are the actual and maximum currents of line $i-j$ respectively,

$N_{G}$ is the set containing all the generators,

$N_{B}$ is the total number of network buses,

$N_{C}$. is the total number of contingencies to be considered,

$\Delta P_{h, d d}^{p}$ is the limit permitted of load curtailment at bus $d$,

$\Delta P_{h, g k}^{p}$ is the ramping limit permitted of generator $k$.

In (1), the objective function is intended to calculate ATC by augmenting the total generation at bus set $S$ of the sending node/area while the load at bus set $R$ of the receiving node/area is enlarged accordingly. For each transaction, the control variables include $P_{g k}(k \in S)$, $P_{l i}(d \in R)$ and $Q_{z m}(m \in M)$, and the generation and loads of all buses not participating in this transaction remain fixed which can be seen from constraints (li)-(1j) above. Another aspect need to be stated is that $P_{t d}(d \in S)$ is also referred to as control variables when it is permitted to curtail after contingency. If not considering corrective actions after contingency, $\Delta P_{h, l d}^{p}$ and $\Delta P_{h, \text {, }}^{p}$ will be referred to zero, which makes the ATC value very conservative and can be illustrated later.

As shown above, the great number of constraints in (1), due to a large number of contingencies considered (and even more so if applied to large systems in stressed operation conditions), makes its solution computationally intensive and even impossible. Benders decomposition method is therefore employed to solve the problem defined in (1).

\section{BENDERS DECOMPOSITION FOR ATC CALCULATION}

\subsection{Benders decomposition}

Benders decomposition breaks down the problem into a master problem and a set of slave subproblems. The subproblems are obtained from the original problem by fixing some of the variables and its solution provides the Lagrange multilpliers associated with the fixed variables. The master problem, derived via the duality theory, makes use of the results from the subproblems to determine whether the fixed variables need modification to be used subsequently in the subproblems again. A relaxation process is adapted to the solution of the master problem by keeping only a very small subset of the constraints or perhaps no constraints. Then the subproblems are tested to see if the solution satisfies the remaining constraints. If so, the solution is optimal since the objective has been minimized over all constraints. If not, the relaxed master is updated by adding the violated constraint and the master problem will be re-solved with more constraints. Iteratively, the master and subproblems interact together until convergence.

It has noticeable advantages as follows:

- It is flexible to use it concurrently with the existing system models and solution procedures.

- The subproblems can be solved independently of each other, and can benefit directly from parallel processing technologies. The method can also deal with unfeasibility of the subproblems.

- The possibility of extending model structural details and features without major changes in the algorithm itself.

- It has strong ability to treat mixed continuous and integer variables. And no linearity assumption is made on the variables.

\subsection{Subproblem definition}

In our study, each contingency case is distinctively defined as an independent subproblem. The constraints of the subproblem include its $p$-th equality and inequality constraints together with the coupling constraints. The objective of the subproblem is to minimize the weighed deviation of the post-contingency 
control from the optimized normal case operating point such that the coupling constraints are satisfied. If the objective function equals to zero or certain tolerance, then a feasible operating point has been reached.

The $p$-th contingency subproblem is defined as $(p=1$, $\left.2, \cdots, N_{C}\right)$ :

$$
\begin{array}{ll} 
& \operatorname{Min} c_{g} \alpha_{p g}+c_{l} a_{p l} \\
\text { s.t. } & \\
& g_{p}\left(x_{p}, u_{p}\right)=0 \\
& h_{p}\left(x_{p}, u_{p}\right) \leq 0 \\
& \bar{u}_{0 l}^{k}-\bar{u}_{p l}-\alpha_{p l} \leq \Delta \bar{u}_{p^{\prime}} \\
& \left|\bar{u}_{0 g}^{k}-\bar{u}_{p g}\right|-\alpha_{p g} \leq \Delta \bar{u}_{p g} \\
& \alpha_{p g} \geq 0 \\
& \alpha_{p l} \geq 0
\end{array}
$$

Where, $c_{g}$ and $c_{l}$ are the two penalty vectors for generation rescheduling and load curtailment, respectively; $\alpha_{p x}$ and $\alpha_{p l}$ are the two vectors that measure the incurred violations associated with the post-contingency control subsets $\bar{u}_{p g}$ and $\bar{u}_{p l}$, respectively; $\bar{u}_{0 g}^{k}$ and $\bar{u}_{0 l}^{k}$ correspond to the two subsets of optimized normal case control vector for the $k$-th iteration, i.e., real power generation and real load demand in the bus sets $S$ and $R$ respectively, which is the information coming from the master problem to the subproblem $p$.

It is obvious that each constraint in (1) can be categorized into one type of constraints in (2). Especially, the coupling constraints $(2 \mathrm{~d})$ and $(2 \mathrm{e})$ correspond to the constraints ( $1 \mathrm{k})$ and (11), respectively.

In the first iteration, $\bar{u}_{0 g}^{k}$ and $\bar{u}_{i j}^{k}$ will be set to the initial optimized normal case power generation of the set $S$ and load demand of the set $R$ (i.e., $\bar{u}_{0 g}^{0}$ and $\bar{u}_{0 l}^{0}$ ). They will be updated in forthcoming iterations marking the new normal case operating point because of the contingency impacts included.

It is obvious that if at the solution point where the objective function equals to zero or is less than the prespecified tolerance (i.e. all the coupling constraints are satisfied), then a feasible solution is obtained for the $p$ th subproblem, which will not impose additional constraints on the master problem. Otherwise, the $p$-th subproblem solution will provide the master problem with violation amounts ( $\alpha_{p g}$ and $\alpha_{p l}$ ) associated with the coupling constraints.

\subsection{The master problem definition}

The two coupling constraint violations $\left(\alpha_{p q}\right.$ and $\alpha_{p l}$ ) for the particular contingency $p$ subproblem will be fed back to the master problem. Consequently, the master problem with the Benders cuts is defined as follows:

$$
\begin{array}{ll} 
& \operatorname{Max} J=\sum_{k \in S} P_{h, G i}\left(x_{0}, u_{0}\right) \\
\text { s.t. } & g_{0}\left(x_{0}, u_{0}\right)=0 \\
& h_{0}\left(x_{0}, u_{0}\right) \leq 0 \\
& \alpha_{g}^{*}+\bar{u}_{k 0}-\bar{u}_{g 0}^{k} \leq 0 \\
& \alpha_{i}^{*}+\bar{u}_{i 0}-\bar{u}_{i 0}^{k} \leq 0
\end{array}
$$

Where, $a_{g}^{*}=a_{p g}, a_{l}^{*}=a_{p l}$. The last two constraints (3d) and (3e) are the Benders cuts turned over from the current studied subproblem to master problem when the subproblem objective is more than the pre-specified tolerance, while contains the information about the impacts of contingency $p$ on the normal case control vector $u_{0}$. It should be pointed out that the initial $u_{0}^{0}$ is solved without the last two constraints (3d) and (3e).

\section{AN IMPROVED PARALLEL SOLUTION SCHEME}

For parallel scheme, all the subproblems associated with different contingencies are processed in parallel, and then Benders cuts from different subproblems are concurrently fed back to the master problem. The master problem will then be solved according to (3) with all the Benders cuts included, which leads to new normal case $u_{0}^{k+1}$. Subproblem (2) for $p=1, \cdots, N_{c}$ should then be solved in parallel under the solved new normal case $u_{0}^{k+1}$. The iteration between the master problem and subproblems ends until no violation occurs for all the subproblems.

As mentioned earlier, if all the Benders cuts are included, it is generally onerous to optimize the master problem with a large number of Benders cuts. Therefore, as shown in Fig.1, an improved parallel scheme, which takes the average value of all non-zero vectors $\alpha_{p}$, and forms only one Benders cut for introducing to master problem, is suggested for the master problem. Since the final ATC solution is based on 'no violation of constraints in all the contingencies', so the solved ATC using the improved parallel solution scheme will be accurate. 


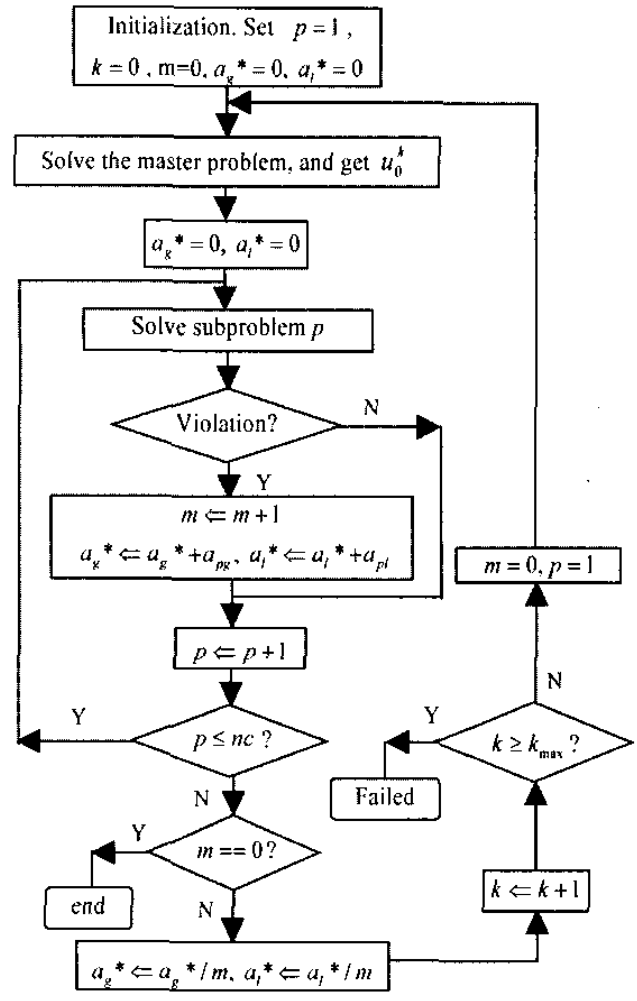

Fig. 1: An improved paralle! scheme for SSC-ATC calculation

\section{NUMERICAL RESULtS}

The presented formulation and solution scheme have been tested on a 2-generator, 4-bus system (see Fig. 2). Four different cases are used to illustrate the effectiveness of the presented method and the necessity of considering post-contingency generation rescheduling and load curtailment. Case 1: from point 2 to point 1; Case 2: from point 3 to point 1; Case3: from point 2 to point 4; Case 4 : from point 3 to point 4 .

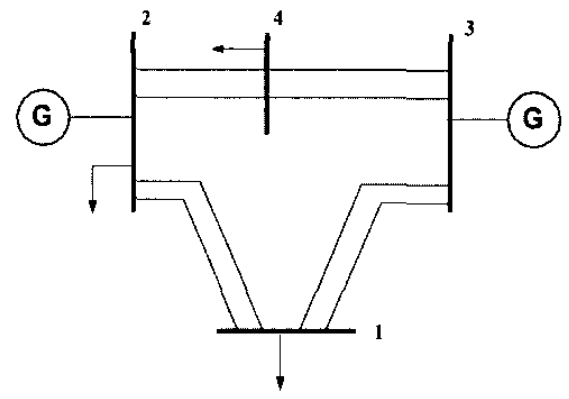

Fig. 2: 4-bus test system

\subsection{Effect of considering ' $\mathrm{N}-1$ ' constraint on ATC}

As shown in Fig.3, the results without considering ' $\mathrm{N}-1$ ' security constraint get too optimistic estimation of ATC in most cases. Therefore, ' $\mathrm{N}-1$ ' security constraint should be considered in evaluating ATC, which, however, make ATC value extremely conservative, which leads to a costly and inefficient use of the network. It is therefore necessary to increase ATC value using post-contingency corrective control.

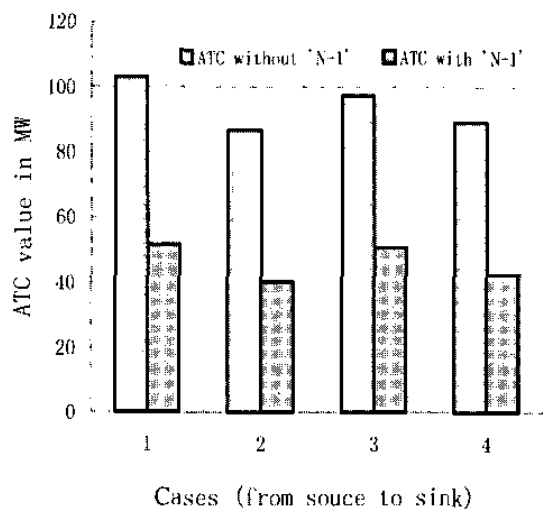

Fig. 3: Effects of considering 'N-l'security constraint

\subsection{Effect of considering corrective control on ATC}

Four corrective cases are considered to illustrate the effects of different corrective controls on ATC values. $\mathrm{S} 1$ : with ' $\mathrm{N}-1$ ' line contingency constraint and without corrective control; $\mathrm{S} 2$ : with generation rescheduling which is permitted to five percent of $P_{h, g k}^{\mathrm{max}} ; \mathrm{S} 3$ : with load curtailment which is permitted to ten percent of $P_{h, l d}^{\max } ; \mathrm{S} 4$ : with both generation rescheduling and load curtailment above.

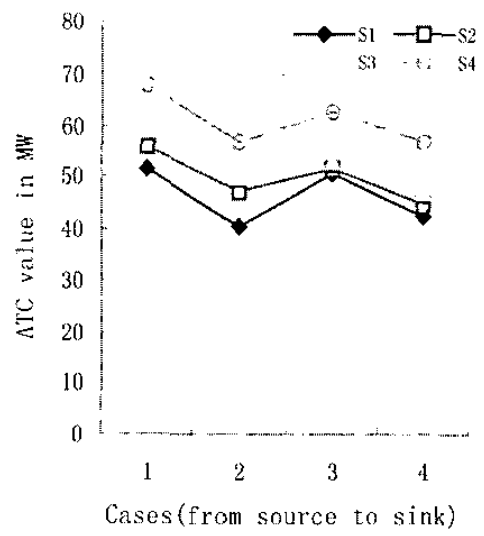

Fig. 4: Effects of considering different corrective controls on ATC 
It can be seen from Fig. 4 that ATC value can be largely increased using post-contingency corrective control and different corrective controls have different effects on it. Their economy can be compared through reliability analysis.

Load curtailment schemes are generally classified into two kinds: called the uniform curtailment scheme and the curtailment scheme based on mathematic programming, respectively. The former can also be reclassified into two subschemes based on either cutting load for all buses by the same amount of power or reducing load for all buses by the same percentage. As compared with the latter, the former, though being more equitable and nondiscriminatory, may result in an excessive and unnecessary reduction of ATC value. As shown in (2a), the latter is therefore employed to illustrate its effect on ATC in this paper. For the latter, all elements in $c_{g}$ and $c_{l}$ should be given different values based on the practical system. In this paper, all elements in $c_{l}$ are given uniform value, which five times the uniform value in $c_{g}$.

\subsection{Comparative study on the two solution schemes}

The solved ATC values for S4 using the improved parallel and conventional serial schemes are shown in Table 1. It is can be seen from the table that the improved parallel and conventional serial schemes get almost the same ATC value, which verifies the improved parallel solution scheme is accurate and effective, therefore be very attractive. As shown in Table 1, the improved parallel scheme is slower than the conventional serial scheme since the former gradually considers all contingencies for any iteration without parallel computer. It can therefore be concluded that using parallel computer, the parallel scheme will be faster; otherwise the serial scheme will be more efficient. Therefore, SSC-ATC calculation based on Benders decomposition should be performed using the corresponding solution schemes according to the computer types.

TABLE 1: ATC VALUE SWITH TWO SOLUTION SCHEMES (IN MW)

\begin{tabular}{c|c|c|c|c|c|c}
\hline Cases & \multicolumn{6}{|c}{ Solution schemes } \\
\cline { 2 - 7 } & \multicolumn{2}{|c|}{ Improved parallel scheme } & \multicolumn{3}{|c}{ Serial scheme } \\
\cline { 2 - 7 } & ATC & $\begin{array}{c}\text { time } \\
(\mathrm{s})\end{array}$ & lterations & ATC & $\begin{array}{c}\text { time } \\
(\mathrm{s})\end{array}$ & Iterations \\
\hline 1 & 66.84 & 2.69 & 3 & 66.87 & 2.17 & 4 \\
\hline 2 & 56.80 & 2.50 & 3 & 56.80 & 1.88 & 3 \\
\hline 3 & 62.76 & 3.46 & 4 & 62.73 & 2.89 & 3 \\
\hline 4 & 56.77 & 3.13 & 3 & 56.78 & 2.74 & 3 \\
\hline
\end{tabular}

\section{Conclusions}

SSC-ATC calculation incorporating post-contingency generation rescheduling and load curtailment was investigated in this paper. Benders decomposition method was successfully used for SSC-ATC calculation. An improved parallel solution scheme has been embedded into the Benders decomposition method to make the calculation more efficient. Computer test results demonstrate the necessity of considering post-contingency corrective control and the effectiveness of the presented method and solution scheme in calculating SSC-ATC.

\section{ACKNOWLEDGEMENTS}

The research is supported by National Key Basic Research Special Fund (No. G1998020305), P.R. China, RGC of Hong Kong government and CRCG of the University of Hong Kong, to whom sincere acknowledgements are expressed.

\section{REFERENCES}

[1] Liu C.C., Heydt, G.T., Edris, A.A.: "Impact of FACTS controller on transfer capability of power grids," in 2002 IEEE Power Engineering Society Winter Meeting, New York, January 2002. pp. 556-561

[2] Armando M.Leite da Silva, Joao Guilherme de Carvaho Costa, Luiz Antonio da fonseca Manso et al.: Andsers. "Transmission capability: availability, maximum transfer and reliability," IEEE Trans. Power Systems, Vol. 17, pp. 843-849, August 2002.

[3] NERC Transmission Transfer Capability Task Force: "Available transfer capability definitions and determination," North Princeton, New Jersey, 1996

[4] Ejebe, G.C., Waight, J.G., Santos-Nieto, M. et al.: "Fast calculation of linear available transfer capability," IEEE Trans. Power Systems, Vol. 15, pp. 1112-1116, August 2000.

[5] Chiang, H.D., Flueck, A.J., Shah K.S. et al.: "CPFLOW: a practical tool for tracing power system steady state stationary behaviour due to load and generation variations," IEEE Trans. Power System, Vol. 10, pp. 623634, May 1995.

[6] Hur, D., Park, J.K., Kim, B.H. et al.: "Security constrained optimal power flow for the evaluation of transmission capability on electric power system," in 2001 IEEE Power Engineering Society Summer Meeting, Vancouver, July 2001. pp. 1133-1138

[7] Geoffrion AM.: Generalized Benders decomposition. $J$. of Optimization theory and Applications 1972; 10(4): 237-260. 